\title{
La comunicación oral en el aula de lengua extranjera
}

\section{Oral communication in the foreign language classroom}

Irene Alonso Aparicio*

Recibido: 22 de febrero de 2012

Aceptado: 24 de mayo de 2012

\section{Resumen}

Como docentes de lengua extranjera, nuestra labor debe consistir, entre otras funciones, en favorecer en el aula las condiciones que desencadenan los procesos y subprocesos implicados en la adquisición lingüística. Algunos de estos procesos incluyen el procesamiento de la lengua meta, la producción lingüística, y la asimilación de información sobre el funcionamiento de lengua meta. La comunicación oral se presenta como marco en el que se despliegan de manera más o menos simultánea dichos procesos y subprocesos. Por ello, por la capacidad de esta actividad para fomentar la adquisición lingüística se reflexiona en este artículo sobre sus posibilidades desde una perspectiva teórica y empírica. Seguidamente, se proponen algunas líneas de actuación didáctica para llevar al aula.

Palabras clave: comunicación oral, lengua extranjera, paradigma cognitivo-interaccionista.

\section{Abstract}

As foreign language teachers, it is our endeavor to favor in the classroom the conditions that trigger the processes and subprocesses involved in language acquisition. Some of these processes include processing the target language, language production, and assimilating information about the target language. Interestingly, oral communication is a potential framework in which such learning processes might more or less simultaneously unfold. Thus, we reflect in this paper on the possibilities of oral communication to promote language learning from a theoretical and empirical stance. Following, a set of teaching guidelines are considered.

Keywords: Oral communication, foreign language, cognitive-interactionist paradigm.

*Doctora con Mención Europea en Didáctica de la Lengua y la Literatura por la Universidad de Granada (España). Profesora Asociada de Español como Lengua Extranjera, Departamento de Español del Centro de Lenguas de la Universidad de Aquisgrán, Alemania. Correo electrónico: alonso-aparicio@sz.rwth-aachen.de 


\section{Introducción}

El aprendizaje de una lengua extranjera (LE) es sin duda un proceso complejo y en el que intervienen muchos subprocesos. Como docentes de LE, es nuestro objetivo crear en el aula las condiciones más favorables posibles para su despliegue. Por esta razón, se reflexiona en este artículo sobre la contribución de algunos de estos procesos al aprendizaje lingüístico y sobre los posibles escenarios para su desencadenamiento en el aula. Más específicamente, se reflexiona sobre la contribución al desarrollo lingüístico del procesamiento de la lengua meta, de la producción lingüística y de la asimilación de información sobre la lengua que se aprende. A continuación se considera cómo puede la comunicación oral fomentar de manera más o menos simultánea el despliegue de estos procesos. Finalmente, se sugieren algunas directrices para la implementación en el aula de la comunicación oral.

\section{Procesos implicados en el aprendizaje lingüístico}

Algunos de los procesos y subprocesos implicados en el aprendizaje lingüístico incluyen el procesamiento de la lengua meta o input ${ }^{1}$ comprensible, la producción lingüística y la asimilación de información sobre el funcionamiento de la lengua meta. La cuestión que entonces surge es: ¿cómo contribuyen estos procesos al desarrollo de la LE? Veamos.

En primer lugar, es indiscutible que la exposición al input es indispensable para que se desencadene la adquisición de una LE. Asimismo, "se da por hecho que sin comprensión, no puede haber aprendizaje" (Gass, 1997, p. 87) (traducción de la autora, en adelante TA). ${ }^{2}$ En otras palabras, el preámbulo para que tenga lugar el aprendizaje es la exposición y procesamiento de muestras comprensibles de la lengua meta -hipótesis del input comprensible- (Krashen, 1985; Long, 1983).

No obstante, con oír y leer no basta. En este sentido, Swain $(1985,1993,1995,1998,2000)$ sostiene que la producción lingüística desempeña asimismo funciones esenciales en el desarrollo de la LE. En primer lugar, propone la investigadora que la producción lingüística estimula al aprendiz a analizar la lengua sintácticamente y no semánticamente como suele ser el caso en la comprensión. Es decir, en la comprensión por ejemplo del enunciado "Los abuelos vienen de visita mañana", es suficiente con un procesamiento semántico para descodificar el significado del enunciado, esto es, basta con conocer el significado de las palabras abuelos, vienen, visita y mañana, no se necesita llevar a cabo un análisis sintáctico sobre la organización de los constituyentes de la oración o la concordancia sujeto-verbo. Sin embargo, en la producción lingüística sí se necesita llevar a cabo dicho análisis sintáctico, esto es, se necesita ordenar los pensamientos en una estructura sintáctica. La producción lingüística puede ser entonces "el desencadenante que fuerza al aprendiz a prestar atención a los medios de expresión necesarios para transmitir con éxito el significado deseado" (Swain, 1985, p. 249) (TA). ${ }^{3}$ Por otro lado, la producción lingüística puede estimular además al aprendiz a captar la diferencia entre lo que quiere

1 Por su difusión en la literatura, para referirnos a las nociones de caudal lingüístico, producción lingüística y parte de datos percibida e incorporada en la memoria a corto plazo, empleamos respectivamente las voces inglesas input, output e intake.

2 "It is a given that without understanding, no learning can take place" (Gass, 1997, p. 87).

3 "the trigger that forces the learner top pay attention to the means of expression needed in order to successfully convey his or her intended meaning."(Swain, 1985, p. 249). 
y puede expresar -noticing the hole- (Swain, 1993, 1995) o entre su interlengua (IL) ${ }^{4}$ y la LE -noticing the gap- (Swain, 1998). Por su parte, esta toma de conciencia de la carencia de recursos puede desencadenar el procesamiento sintáctico de un input posterior a fin de encontrar los recursos necesarios, y la formulación de hipótesis sobre el funcionamiento de la LE. Más aún, la formulación de hipótesis sobre el funcionamiento de la LE puede además desempeñar una función metalingüística (Swain, 1998, 2000), es decir, puede invitar al aprendiz a formular hipótesis sobre el funcionamiento de la lengua meta, convirtiéndose esa formulación en objeto de reflexión y su producto en conocimiento aprendido. $\mathrm{O}$ lo que es lo mismo, la producción lingüística puede servir como herramienta para reflexionar sobre la lengua. Por otro lado, una vez formulada una hipótesis sobre el funcionamiento de la LE (metalingüística o no), la producción lingüística puede además convertirse en el escenario en el que comprobarla (verificarla o rechazarla), especialmente cuando se obtiene retroalimentación externa (Swain, 1993, 1995). Es decir, la producción puede desencadenar la comprobación de hipótesis y la provisión de retroalimentación. Por último, Swain $(1985,1993,1995)$ reconoce además la capacidad de la producción lingüística para contribuir a la automatización de una forma meta cuando se lleva a cabo de manera reiterada.

Sin embargo, comprensión y producción siguen sin bastar y un ejemplo de ello lo encontramos en la evaluación de la implantación de los enfoques comunicativos en los programas canadienses de educación obligatoria en francés/LE, pues dicha evaluación revela que tras una media de siete años de exposición al francés, los estudiantes no consiguen producir correctamente algunos aspectos morfológicos y sintácticos (por ejemplo, la morfología verbal) (Swain, 1985). Por otro lado, el aprendizaje en el contexto natural presenta también ciertas limitaciones. Un ejemplo de ello lo constituyen los trabajos de Schmidt (1983) y Sato (1990), cuyos sujetos, pese a desarrollar la LE en el contexto natural durante amplios periodos muestran unos niveles de competencia gramatical considerablemente inferiores a los presentados en el examen de la competencia, por ejemplo, discursiva. Concretamente, presentan notables dificultades con la producción del morfema de pasado -ed en inglés/LE, una forma que curiosamente se domina con relativa rapidez en el contexto de aula con instrucción formal (Bardovi-Harlig, 1995; Doughty y Varela, 1998; R. Ellis et ál., 2006). Una explicación potencial a estos fenómenos se encuentra en la hipótesis de la captación (Schmidt, 1990, 1994). De acuerdo con esta hipótesis, la captación o registro consciente de una forma en la memoria a corto plazo "es la condición necesaria y suficiente para la conversión del input en intake en la adquisición de la segunda lengua" (Schmidt, 1994, p. 176) (TA). ${ }^{5}$ Es decir, se puede argüir entonces que los aprendices de los casos previamente mencionados no han aprendido ciertas formas porque no las han captado. Por otro lado, White $(1989,1991)$ sostiene que la exposición a un input comprensible también puede resultar insuficiente para el desarrollo correcto de algunas formas cuando las estrategias naturales de aprendizaje resultan contraproducentes o insuficientes para la formulación de hipótesis correctas sobre su funcionamiento. ${ }^{6}$ Para promover entonces la captación de formas que puedan pasar desapercibidas o que puedan simplemente no adquirirse debido a las

4 El término interlengua (IL) (Selinker, 1972) hace referencia al sistema lingüístico autónomo que configura el aprendiz en su intento de producir la norma de la lengua meta.

5 "is the necessary and sufficient condition for the conversion of input to intake in SLA". (Schmidt, 1994, p. 176)

6 El término interlengua (IL) (Selinker, 1972) hace referencia al sistema lingüístico autónomo que configura el aprendiz en su intento de producir la norma de la lengua meta. 
estrategias naturales de procesamiento de la información, se propone a lo largo de los noventa del pasado siglo la necesidad de que el aprendiz sea provisto de información explícita sobre el funcionamiento de la lengua meta. ${ }^{7}$ Surge entonces el concepto de atención a la forma (Long, 1991; Long y Robinson, 1998). Esta expresión alude a una intervención pedagógica que ocasionalmente y de forma breve dirige la atención del estudiante a los rasgos formales del código cuando una necesidad o dificultad comunicativa así lo exige.

A la luz entonces de estos procesos implicados en el aprendizaje lingüístico, la cuestión que surge es, ¿de qué manera puede el docente de LE desencadenarlos en el aula? Abordamos esta cuestión en los apartados que se suceden.

\section{Contribución de la comunicación oral: planteamientos teóricos y datos empíricos}

Inspirado en los procesos identificados en el aprendizaje lingüístico, Long $(1983,1996,2007)$ postula en su hipótesis de la interacción a comienzos de los ochenta del pasado siglo y a lo largo de las décadas posteriores, que la negociación de significado que tiene lugar en la interacción comunicativa entre el hablante no nativo (HNN) y el hablante nativo (HN) o el HNN con mayor dominio de la lengua extranjera, facilita la adquisición lingüística porque favorece el despliegue de procesos implicados en el aprendizaje de la lengua meta: (a) la provisión de lengua o input comprensible; (b) la oportunidad de producción lingüística; y (c) la asimilación de información sobre el funcionamiento de la lengua meta. Más específicamente, propone Long que:
La negociación de significado, y especialmente la ne- gociación que desencadena ajustes en la interacción por parte del HN o de un interlocutor más compe- tente, facilita la adquisición porque conecta de for- ma productiva el input, las capacidades internas del aprendiz, una atención particularmente selectiva, y el output. (Long, 1996, p. 451-452) (TA) ${ }^{8}$

En efecto, la investigación ha demostrado que los ajustes conversacionales -estrategias y tácticas que evitan y reparan respectivamente fallos en la comunicación- que tienen lugar durante la negociación de significado proporcionan un input más comprensible al aprendiz (Long, 1983). Considérese en este sentido el siguiente ejemplo (1), extraído de Mackey (1999, pp. 558-559) (TA): ${ }^{9}$

$\mathrm{HN}$ : Hay hay un un par de gafas de lectura sobre la planta.

HNN: ¿Un qué?

HN: Gafas ¿gafas de lectura para leer el periódico?

HNN: ¿Gafis?

$\mathrm{HN}$ : Te las pones para ver, si no puedes ver. Gafas de lectura.

7 Véase Norris y Ortega (2000) para un meta-análisis sobre las posibilidades de proporcionar información más o menos explícita al aprendiz sobre el funcionamiento de la lengua meta.

8 "negotiation for meaning, and especially negotiation work that triggers interactional adjustments by the NS or more competent interlocutor, facilitates acquisition because it connects input, internal learner capacities, particularly selective attention, and output in productive ways." (Long, 1996, pp. 451-452).

9 “NS: There's there's a apair of reading glasses above the plant. NNS: A what? NS: Glasses reading glasses to see the newspaper? NNS: Glassi? NS: You wear them to see with, if you can't see. Reading glasses. NNS: Ahh ahh glasses glasses to read you say reading glasses. NS: Yeah" (Mackey, 1999, pp. 558-559). 
HNN: Ahh ahh gafas gafas para leer se dice gafas de lectura.

\section{HN: Sí.}

Como se observa, la locución gafas de lectura ocasiona un problema de comprensión al HNN. Se desencadena entonces un proceso de negociación de su significado entre el HN y el HNN en forma de peticiones de aclaración y confirmación por parte del HNN, y de reformulaciones por parte del HN. El resultado de esta negociación del significado es la comprensión por parte del $\mathrm{HNN}$ de la expresión que en un principio le había ocasionado cierta perplejidad.

Por otro lado, "la retroalimentación negativa [...] puede facilitar el desarrollo de la L2" (Long, 1996, p. 414) (TA $)^{10}$. Es decir, los ajustes conversacionales desencadenados tras la incorrección de la locución emitida por el HNN pueden proporcionar a este información explícita (por ejemplo, mediante una regla metalingüística) o implícita (por ejemplo, mediante la reformulación de su locución) sobre su incorrección. Idílicamente, esta información llevará al HNN a prestar atención a su incorrección lingüística sin desviarse de su intención comunicativa (dado que su atención está centrada en la significación del acto comunicativo), pero a la misma vez, sin necesidad de reparar en el significado comunicativo de la corrección porque ya lo conoce (dado que supone una reformulación de su propia locución). Considérese en este sentido, este otro ejemplo (2) de Mackey y Philp (1998, p. 353) (TA): ${ }^{11}$
HNN: y ah en tu foto ¿niños jugando?

HN: ¿están los niños jugando? Sí

HNN: sí y ¿están contentos o tristes?

HN: están contentos (.) están jugando con la pelota

HNN: y ah ¿están jugando en el jardín cerca de la casa?

HN: sí

Como se observa, el HN proporciona retroalimentación negativa implícita en forma de reformulación al HNN, es decir, reproduce en una versión lingüísticamente correcta, el contenido de su enunciado lingüísticamente incorrecto. El HNN, por su parte, tal como muestran sus dos intervenciones posteriores, parece percibir su incorrección dado que modifica su output y formula correctamente las preguntas requeridas por la tarea que realiza.

Además, como expone Swain (1985, 1993, 1995, 1998), la comunicación oral se constituye como marco que posibilita al aprendiz producir un output empujado o forzado que le permite emplear recursos más adecuados o despertar en él la necesidad de desarrollarlos. Obsérvese el ejemplo (3), extraído de Mackey (1999, p. 559) (TA): ${ }^{12}$

HNN: Y una ar ar más esta foto.

$\mathrm{HN}: \gtreqless E h ?$

10 "Negative feedback [...] may be facilitative of L2 development" (Long, 1996, p. 414).

11 "NNS: and ah in your picture they children playing? NS: are the children playing? NNS: yes and are they happy or sad? NS: They are happy (.) they're playing with the ball. NSS: and ah are they playing in the garden near house? NS: yes". (Mackey y Philp, 1998, p. 353).

12 "NNS: And one more weep weep this picture. NS: Huh? NNS: Another one like gun to shoot them weep weepon. NS: Oh ok ok yeah I don't have a second weapon though so that's another difference" (Mackey, 1999, p. 559). 
HNN: Otra como pistola para dispararles ar arme

HN: Oh vale vale sí. No tengo una segunda arma aunque ésa es otra diferencia.

En este caso, la perplejidad del $\mathrm{HN}$ ante el enunciado incomprensible del HNN empuja a este último a reformular su output y modificarlo para facilitarle al HN su comprensión, un esfuerzo que dada la respuesta del HN, desarrolla con éxito.

Finalmente, la comunicación oral se postula también como escenario en el que el aprendiz someta a evaluación y a continuación, verifique o rechace sus hipótesis sobre la LE. Considérese en este sentido el siguiente ejemplo (4), extraído de Gass y Varonis (1989, pp. 80-81) (TA): ${ }^{13}$

Hiroko: Ah, el perro está ladrando para-

Izumi: A

Hiroko: A la mujer.

En (4), Hiroko parece someter a evaluación una hipótesis sobre la preposición que rige el verbo ladrar en inglés. Como puede observarse, ante la incorrección de la preposición empleada, Izumi llama su atención y le proporciona la versión correcta. Hiroko capta la corrección, rechaza su hipótesis, y adopta la preposición correcta.

\section{Contribución de la comunicación oral: consideraciones metodológicas}

Desde su formulación implícita en 1983, la hipótesis de la interacción suscita gran interés entre la comunidad investigadora (Alcón Soler, 2001; Gass, 1997; Gass, 2003; Gass y Selinker, 1994; Long, 2007); no obstante, su investigación no está exenta de dificultades metodológicas. En este sentido, apuntan Gass y Mackey (2007) la dificultad de identificar datos que evidencien el conocimiento aprendido. Es decir, ante por ejemplo la reformulación que realiza el $\mathrm{HN}$ de su locución y la reproducción que a continuación lleva a cabo el HNN, ¿̇se puede concluir que exista comprensión por parte del HNN o se trata de una imitación sin comprensión como estrategia para salvar la comunicación?, ¿evidencia la repetición del HNN un proceso consciente de comparación de estructuras (la que él hubiera empleado y la vernácula)? Para salvar estas dificultades, proponen las investigadoras complementar la transcripción de las interacciones conversacionales con otros procedimientos de recogida de datos como los pretests y postests, o los juicios introspectivos y retrospectivos. Long (2007) añade además la necesidad de seleccionar la muestra experimental con el objeto de asegurar su desconocimiento lingüístico de las formas meta antes de realizar cualquier investigación con el fin de poder entonces atribuir cualquier atisbo de aprendizaje a la interacción comunicativa.

Sin embargo, pese a estas dificultades y para el caso concreto de las reformulaciones correctivas, Long (2007) ha identificado recientemente en la literatura hasta sesenta estudios descriptivos, cuasidescriptivos y experimentales acerca de su existencia y utilidad en el contexto formal y natural. Tras una revisión parcial pero representativa de la muestra empírica que emplea instrumentos de recogida de datos fiables, Long concluye que: a) las reformulaciones existen en una frecuencia relativamente alta en el contexto formal y natural; b) los aprendices captan la retroalimentación negativa contenida en ellas; c) esta retroalimentación es además utilizable y, en ocasiones, utilizada; y, d) si bien no son estrictamente necesarias para la adquisición, las reformulaciones correctivas sí parecen facilitar el desarrollo de la lengua extranjera.

13 "Ah, the dog is barking to- Izumi: at. Hiroko: at the woman" (Gass y Varonis, 1989, pp. 80-81). 


\section{Implicaciones didácticas}

La hipótesis de la interacción surge de una preocupación psicolingüística, no didáctica. Ahora bien, como de cualquier hipótesis, modelo, o teoría de adquisición que se precie, se pueden extraer unas líneas de actuación pedagógica. De hecho, Long (1996) sugiere en las últimas líneas de su presentación que, partiendo de que su propuesta es una hipótesis y no una premisa consolidada, su postulado insinúa la importancia de:

...tareas que estimulen la negociación de significado $[\ldots]$ porque pueden resultar una de las vías más sencillas para facilitar que el aprendiz se centre en la forma sin perder de vista el foco predominante de una lección (o conversación) en el significado. (Long, 1996, p. 454) (TA) $)^{14}$

En esta misma línea y en lo que a la provisión de retroalimentación negativa se refiere, Long (2007) subraya el potencial didáctico de las reformulaciones, pues dada su naturaleza implícita, le permiten al estudiante reparar en su error sin perder de vista el contenido de la tarea que le ocupa. Asimismo, puesto que es una vía de corrección reactiva -tras producirse el error- y no proactiva -que se anticipa al error-, la reformulación tiene cabida en las ansiadas metodologías centradas en el alumno y no en el profesor.

Por otro lado, tras un análisis pormenorizado de la investigación centrada en los factores que favorecen o inhiben la negociación, Gass (1997) concluye que tareas en pequeños grupos o en parejas, tareas con un objetivo común (por ejemplo, la resolución de un problema), tareas de vacíos de información (como el dictado gráfico), y tareas que requieren el intercambio obligatorio de información y el con- senso hacia una única solución posible (como ordenar una secuencia), propician más la negociación que el discurso entre profesor y alumnado, y que la conversación libre y/o espontánea (por ejemplo, un debate). Por el contrario, una diferencia considerable en el estatus lingüístico y el conocimiento previo de los interlocutores parece inhibirla. Asimismo, la falta de familiaridad o el desconocimiento mutuo entre los interlocutores inhibe la negociación. Finalmente, la variable género, si bien poco documentada, parece impactar también en la cantidad y calidad de la negociación, propiciando la interacción entre interlocutores del mismo género y (más la negociación que la interacción entre interlocutores de distinto género), cuando ambos son hablantes no nativos.

Por último, además de lo ya expuesto, Vázquez Bourgon (2003) añade que la interacción en pequeños grupos fomenta la reducción de la ansiedad e incrementa la autoestima, la motivación, la autonomía de aprendizaje y la autodeterminación.

\section{Conclusiones}

De la misma manera que intuimos que a andar se aprende andando, intuimos que a hablar en la lengua meta se aprende hablando. El objetivo de este artículo ha sido reflexionar sobre esta intuición e intentar describirla científicamente desde el paradigma cognitivo-interaccionista. En este sentido y siguiendo a Gass y Mackey, concluimos que la comunicación oral puede facilitar el desarrollo de la IL:

...porque proporciona al aprendiz la oportunidad de recibir input modificado y de recibir feedback, tanto explícitamente como implícitamente, lo que a su vez puede dirigir su atención

14 "tasks that stimulate negotiation for meaning [...] for they may be one of the easiest ways to facilitate a learner's focus on form without losing sight of a lesson's (or conversation's) predominant focus on meaning” (Long, 1996, p. 454). 
a aspectos problemáticos de su interlengua y forzarle a producir un output modificado. (Gass y Mackey, 2007, p. 194) (TA) ${ }^{15}$

Asimismo, hemos recogido en estas líneas los principales hallazgos de este paradigma de investigación y reflexionado sobre la explotación didáctica de la comunicación oral en el aula de lenguas extranjeras. Los resultados son prometedores y la inclusión en el aula de tareas que fomenten la interacción comunicativa, siempre que estén cuidadosamente diseñadas, se presenta como una práctica imprescindible para el docente, tanto por sus ventajas psicolingüísticas, como por su flexibilidad para adaptarse a los estilos de aprendizaje, preferencias y características individuales de los estudiantes. Por todo ello, se subraya desde aquí la necesidad de fomentar la comunicación oral en los procesos de enseñanza y aprendizaje de lenguas extranjeras.

\section{Referencias}

Alcón, E. (2001). Interacción y aprendizaje de segundas lenguas en el contexto institucional del aula. Revista Estudios Lingüísticos de la Universidad de Alicante, Anexo 1, 271- 287.

Gass, S. (1997). Input, Interaction, and the Second Language Learner. Mahwah, NJ: Lawrence Erlbaum Associates.

Gass, S. (2003). Input and Interaction. En C. Doughty y M. Long (Eds.). The Handbook of Second Language Acquisition (pp. 224-255). MA: Blackwell Publishing.
Gass, S. y Mackey, A. (2007). Input, Interaction, and Output in Second Language Acquisition. En B. Vanpatten y J. Williams (Eds.). Theories in Second Language Acquisition. An Introduction (pp. 175-199). Mahwah, NJ: Lawrence Erlbaum Associates.

Gass, S., Mackey, A. y Pica, T. (1998). The Role of Input and Interaction in Second Language Acquisition. The Modern Language Journal, 82, 299-305.

Gass, S. y Selinker, L. (1994). Second Language Acquisition. An Introductory Course. Mahwah, NJ: Lawrence Erlbaum Associates.

Gass, S. y Varonis, E. (1989). Incorporated repairs in NNS discourse. En M. Eisenstein (Ed.).The dynamic interlanguage (pp. 71-86). New York: Plenum.

Krashen, S. (1985).The Input Hypothesis: Issues and Implications. New York: Longman.

Long, M. (1983).Native speaker/non-native speaker conversation and the negotiation of comprehensible input. Applied Linguistics, 4 (2), 126-141.

Long, M. (1991). Focus on form: A design feature in language teaching methodology. En K. de Bot, R. Ginsberg y C. Kramsch (Eds.). Foreign language research in cross-cultural perspective (pp. 39-52). Amsterdam: John Benjamins.

Long, M. (1996). The Role of the Linguistic Environment in Second Language Acquisition. En W. Rithie y T. Bhatia (Eds.). Handbook of Second Language Acquisition (pp. 413-468). San Diego: Academic Press.

15 "as it provides learners with opportunities to receive modified input and to receive feedback, both explicitly and implicitly, which in turn may draw learners' attention to problematic aspects of their interlanguage and push them to produce modified output". (Gass y Mackey, 2007, p. 194) 
Long, M. (2007). Problems in SLA. Mahwah, NJ: Lawrence Erlbaum Associates.

Long, M. y Robinson, P. (1998). Focus on form: Theory, research, and practice. En C. Doughty \& J. Williams (Eds.). Focus on Form in Classroom Second Language Acquisition (pp. 15-41). Cambridge: Cambridge University Press.

Mackey, A. (1999). Input, Interaction, and Second Language Development. An Empirical Study of Question Formation in ESL. Studies in Second Language Acquisition, 21, 557-587.

Mackey, A. y Philp, J. (1998). Conversational Interaction and Second Language Development: Recasts, Responses, and Red Herrings? The Modern Language Journal, 82, 338-356.

Norris, J. y Ortega, L. (2000). Effectiveness of L2 Instruction: A Research Synthesis and Quantitative Metaanalysis. Language Learning, 50 (3), 417-528.

Ortega, L. (2001). Atención implícita hacia la forma: teoría e investigación. Estudios Lingüísticos de la Universidad de Alicante, Anexo 1, 179-212.

Schmidt, R. (1990). The role of consciousness in second language learning. Applied Linguistics, 11 (2), 17-46.

Schmidt, R. (1994). Implicit learning and the cognitive unconscious: Of artificial grammars and SLA. En N. Ellis (Ed.). Implicit and explicit learning of languages (pp. 165-209). London: Academic Press.

Selinker, L.(1972). Interlanguage. International Review of Applied Linguistics, 10, 209-231.

Swain, M. (1985). Communicative competence: some roles of comprehensible input and com- prehensible output in its development. En S. Gass y C. Madden (Eds.). Input in second language acquisition (pp. 235-253). Rowley, MA: Newbury House.

Swain, M. (1993).The Output Hypothesis: Just Speaking and Writing Aren't Enough. The Canadian Modern Language Review, 50 (1), 158-164.

Swain, M.(1995). Three functions of output in second language learning. En G. Cook y B. Seidlhofer (Eds.). Principle and practice in applied linguistics: Studies in honour of H.G. Widdowson (pp." 125-144). Oxford: Oxford University Press.

Swain, M. (1998). Focus on form through conscious reflection. En C. Doughty y J. Williams (Eds.). Focus on Form in Classroom Second Language Acquisition (pp. 64-81). Cambridge: Cambridge University Press.

Van Patten, B. (1996). Input Processing and Grammar Instruction: Theory and Research. Westport, CT: Ablex.

Vázquez, E. (2003).Fundamentos del trabajo en pequeños grupos o en parejas en la enseñanza de español como lengua extranjera. Revista Cervantes, 6, 37-41.

White, L. (1989). The principle of adjacency in second language acquisition: Do L2 learners observe the subset principle? En S. Gass y J. Schachter (Eds.). Linguistic perspectives on second language acquisition (pp. 134-158). Cambridge: Cambridge University Press.

White, L.(1991).Adverb placement in second language acquisition: some effects of positive and negative evidence in the classroom. Second Language Research, 7, 133-161. 